\title{
Artificial Imagination Induced by Visualised Hypotheses in Archaeology
}

\author{
Dominik Lengyel \\ BTU Cottbus-Senftenberg \\ Konrad-Wachsmann-Allee 4 \\ 03046 Cottbus, Germany \\ lengyel@b-tu.de
}

\author{
Catherine Toulouse \\ BTU Cottbus-Senftenberg \\ Konrad-Wachsmann-Allee 4 \\ 03046 Cottbus, Germany \\ toulouse@b-tu.de
}

\begin{abstract}
This paper demonstrates our 'visualisation of hypotheses' approach for providing scientific and creative visualisations. Our aim is to project the abstract virtual model as realistically as possible, using the technique we call 'virtual photography'. We have developed research projects in close contact with the German Archaeological Institute DAl (antic metropolis Pergamon in West Anatolia, Palatine Palaces in Rome, Ktesiphon in Mesopotamia, all exhibited in the Pergamon Museum Berlin, developed within the Excellence Cluster TOPOI by Freie Universität and HumboldtUniversität Berlin and funded by the German Research Foundation DFG); the Egyptian Museums Berlin and Munich (royal city of Naga, Sudan); Cologne Cathedral (building phases including predecessors); the Martin-von-Wagner Museum Würzburg (visualising Archbishop Julius von Echter's ideal church); and the Bern Minster Foundation and Bern Institute for Art History (the early building phases of the 15th and 16th century). This paper demonstrates how virtual modelling and virtual photography work hand-in-hand, respecting traditional architectural design modelling in order to translate hypotheses and uncertain knowledge, without adding more content than necessary, to induce an architectural vision. In addition, we also follow the design principles of traditional architectural photography. Traditional methods of representing architecture are therefore used in combination with high-end technological tools for creating familiar visual impressions. The benefit of this approach is that the main subject of the examination will be its content, despite its technological approach and appearance. Using the CAD tools of mechanical engineering, which allow geometrical definitions that go far beyond the architectural needs, and visualisation tools that approach the quality of simulations, the visualised hypotheses resemble studio photography of clay models, or realistic photography of abstract geometry.
\end{abstract}

Architecture. Archaeology. Art history. Visualisation. Hypotheses. Uncertainty. Knowledge.

\section{INTRODUCTION}

Hypotheses about architecture in archaeology, building research and art history generally describe a fragmentary vision of the past. This is mainly due to the fact that on a scientific basis there will be no certainty about all aspects of the past. Hypotheses are based on conclusions and analogies, and can be quite diverse or even contradictory. We call this characteristic of science 'uncertain knowledge'. It is precisely this uncertainty, which, as the essence of scientificity, meets with scepticism among the general public, who often expect unambiguous, if not simple, answers. Therefore, the uncertainty in knowledge is not only a concern in communication within the sciences. It is also a concern for us as scientists to bring the uncertainty as a fundamental part of science to the public. To visualise hypotheses about architecture is the consequent translation of hypotheses from verbal to pictorial form. The simplest way to do this would be the diagram, which in the case of architecture also includes coded representations such as ground plans, sections and views. They can contain a considerable amount of information, but rarely provide a spatial impression. Architecture, however, is first and foremost space, and ground plans, sections and elevations have established themselves as a communication tool especially for the construction of architecture. By comparison, sketches and models have traditionally been the primary way of presenting architecture. Hypotheses about lost architecture are formulated in a similar way to the demands on a building to be constructed. In architectural design, essential elements are formulated, but many details are left open. In a hypothesis, essential elements are often also outlined, whereas most details will likely remain hidden forever. This inner, non-resolvable uncertainty fundamentally distinguishes the visualisation of hypotheses from reconstructions. 

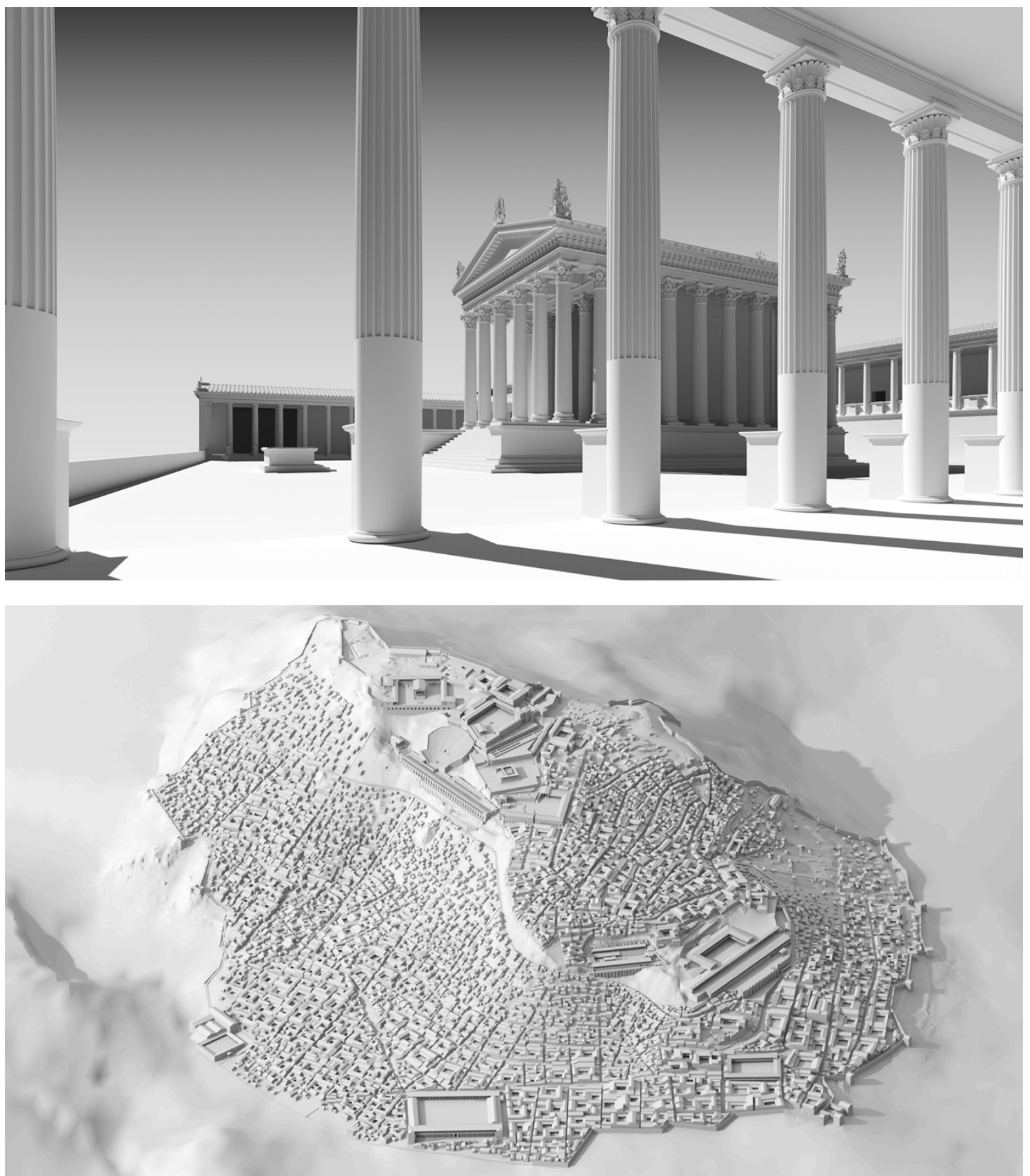

Figure 1: Pergamon around 200 n. Chr. for the German Archaeological Institute.

A reconstruction in the strict sense is a rebuilding of a lost structure. The term assumes that a reconstruction would be at all possible. This assumes that all components of the building were known. This is not given in most cases of hypotheses of the above-mentioned sciences. Therefore, the possibility of a reconstruction in the strict sense of the term is not given. Of course, it would be possible, for example, to construct a building based on a scientifically founded cubature. However, the multitude of necessary constructional decisions alone excludes the term reconstruction in the strict sense. Therefore, we do not speak of a $3 \mathrm{D}$ reconstruction or a virtual reconstruction, but of the visualisation of hypotheses. This term allows any degree of uncertainty to be represented, from a precise rebuilding true to the original to a completely vague indication of a volume.

Reconstructions are however not only difficult on a theoretical level. Above all, they bear the potential cost of transporting certainties that lack any foundation. The problem is a contradiction that cannot be resolved. A reconstruction always pursues a state similar to the original. It seeks to represent that which is lost as something, which has been regained. This main intention of a reconstruction prevents a critical debate on the reconstruction. The visitor, or even just the observer of a reconstruction, will not be aware of 

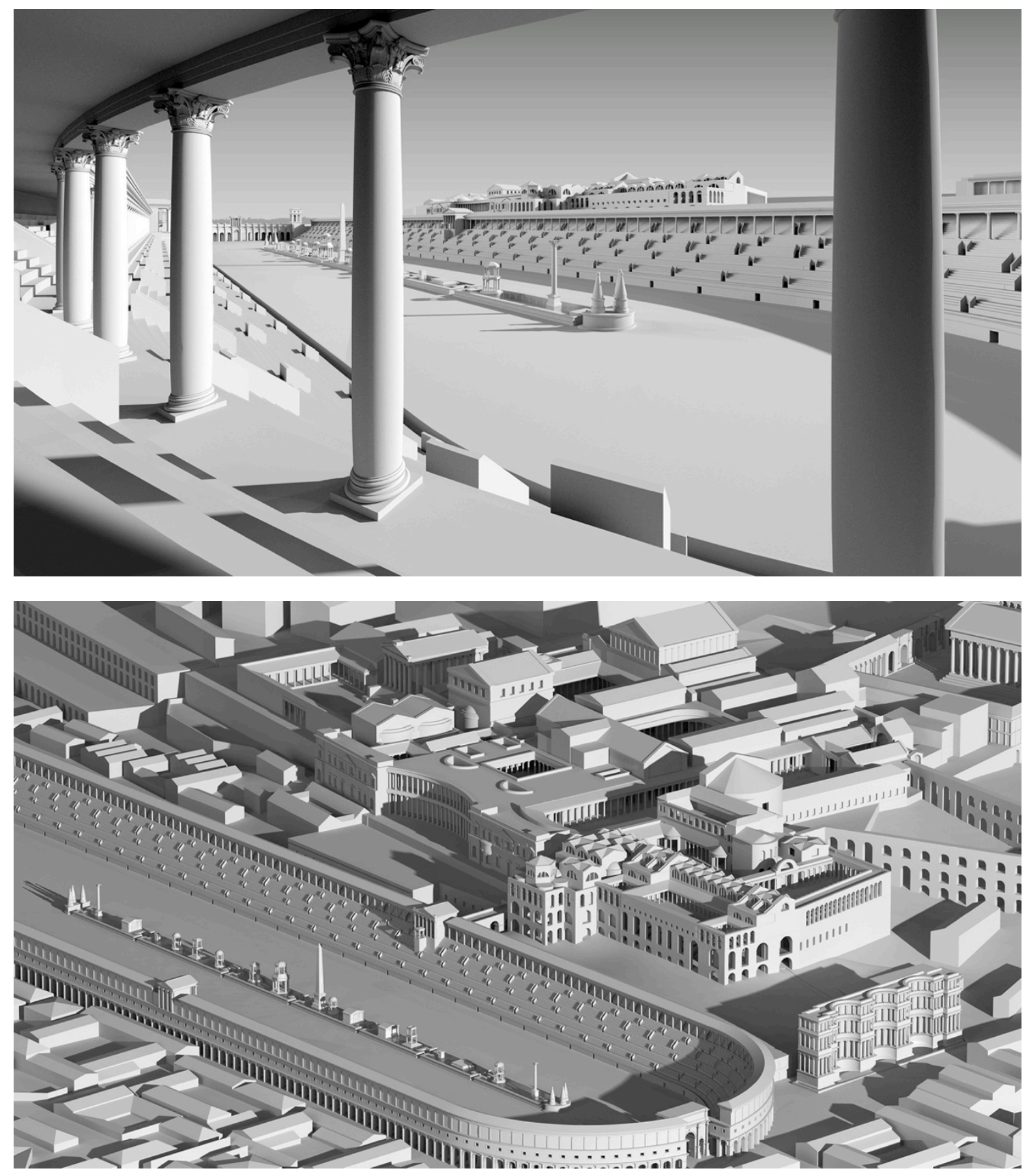

Figure 2: The Palatine Palaces in Rome for the German Archaeological Institute.

any uncertainties, since uncertainties were of course not present in the original building.

The mere visualisation of hypotheses, on the other hand, completely dispenses with this difficulty, since its main purpose is to translate the verbal hypotheses into visual form, regardless of their inner uncertainty or contradictions. The interpretation of the visualisation therefore does not have to deal with the actual reality of the physical environment, but must be measured against the interpretation of the verbal hypothesis. The same applies to the validation of the visualisation, since it is generally the author of the verbal hypothesis who is in charge of it. This fact also keeps the visualisation tightly connected to science. And thus it not only represents uncertain knowledge itself, but also uncertainty as a phenomenon. In this way, the essence of science is conveyed at the same time as the content, thereby promoting an appreciation of scientificity.

There is another level of insight, which lies behind the visualisation of hypotheses. Verbal hypotheses about lost architecture typically describe architecture in a way that emphasises its essential characteristics. Even findings are included in a way that their structural involvement is emphasised. The result is a description similar to that of a building project. Here too, in the process of designing $a$ 

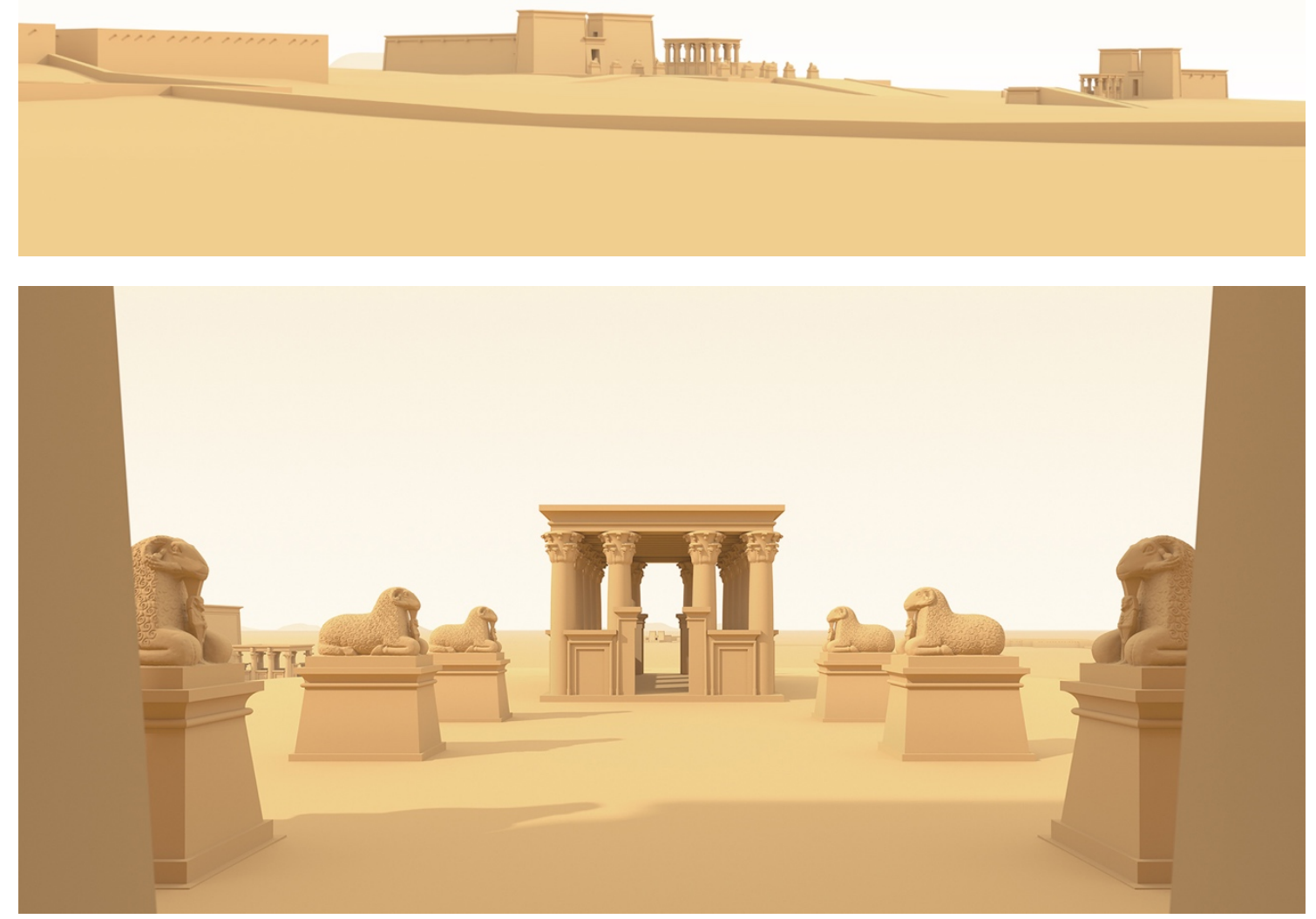

Figure 3: The royal city Naga in today's Sudan for the Egyptian museums in Munich and Berlin.

building, essential characteristics of the future building are negotiated, while many details are left undefined.

The verbal hypothesis often formally corresponds to a fragmentary description of a building plan or architectural design. The visualisation thereby departs even further from a reconstruction, for both the verbal hypothesis and the translated visualisation do not represent the lost building itself, but rather its hypothetical design, or even more extensively, the design intention. In general, this design intention should not be confused with a realised building, but rather a more or less clear abstraction of the same.

\section{ABSTRACTION}

Abstraction is the key term for any form of scientific debate. In the case of visualised hypotheses, the specific meaning of abstraction in a visual context is an important factor. As mentioned above, abstraction is mainly used in diagrams, but it goes much further. Abstraction becomes significant for the visualisation of uncertainty when it avoids unambiguous references such as a picture caption and instead conveys the uncertainty in a subtle way.

Subtlety plays a very important role in the visual arts. From the recipient's point of view, Kasimir Malevich's Black Square already consists primarily of a projective surface that allows infinite interpretations. The more concrete a work of art is, the more the interpretation can be directed. Landscape installations such as those by Donald Judd can easily be interpreted as architecture. One of the prisms from Hubert Kiecol's artwork Three Streets is already almost necessarily interpreted as a building.

Abstraction is also established in the representation of architecture. Geometrically simplified architectural models can already be found in ancient Egypt. And since the Renaissance at the latest, large-scale wooden models have been on hand that convey a purely geometric, if scaled down, image of prospective architecture. What all these models have in common is that although they suggest many things, they also omit others. This applies not only to formal details or, in the vast 

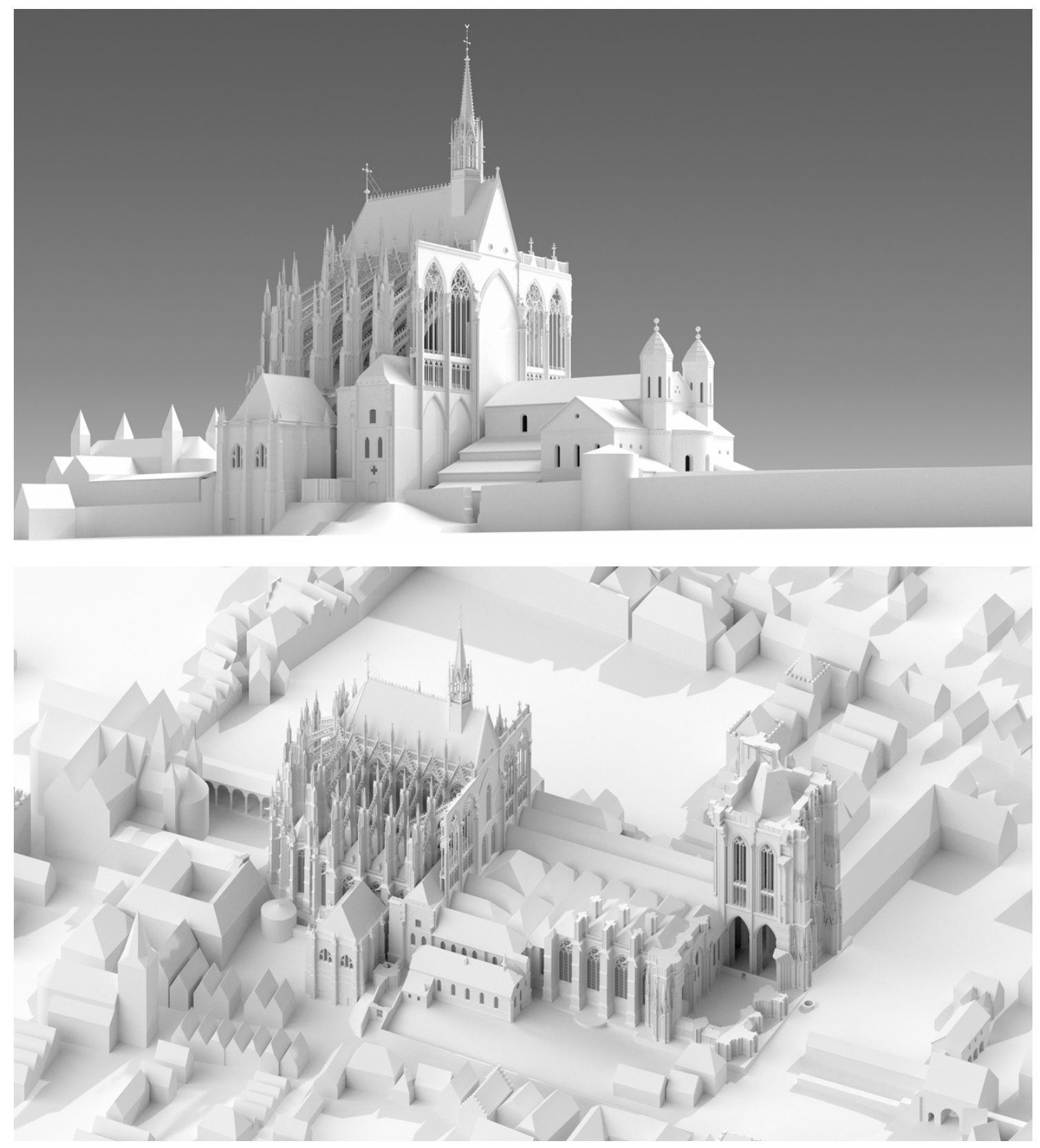

Figure 4: Cologne Cathedral around 1320 (above) and 1540 (below) for the cathedral administration.

majority of cases, the building's construction, but also to its materiality. This becomes particularly obvious when the visible materiality clearly deviates from every possible form of implementation, as was the case with historical cork and plaster models. While in some cases wood can give an impression at least for the interior panelling of representative buildings that may come close to the realisation, this is not the case with cork and plaster models. Here the question arises as to the significance of what would actually be visible in relation to the communicated architecture. Plaster models in particular are clearly limited here to the pure mediation of geometry. It should be considered that this restriction is to be understood more as a concentration, since the absence of materiality conversely makes the plasticity all the more evident. It is therefore by no means a disadvantage to concentrate the representation on the geometry, if this makes the understanding of the spatial structure appear so much clearer.

Abstraction is the focused communication of selected aspects. This implies that the entire image of architecture only materialises in the imagination of the spectator. It is therefore of particular importance in which way the visualisation suggests architecture. Here, too, the diagram would be a possibility. Yet the usual form of unambiguous encoding, often accompanied by an unambiguous legend, is an attempt to inform as unambiguously as possible about the degree of uncertainty, for 

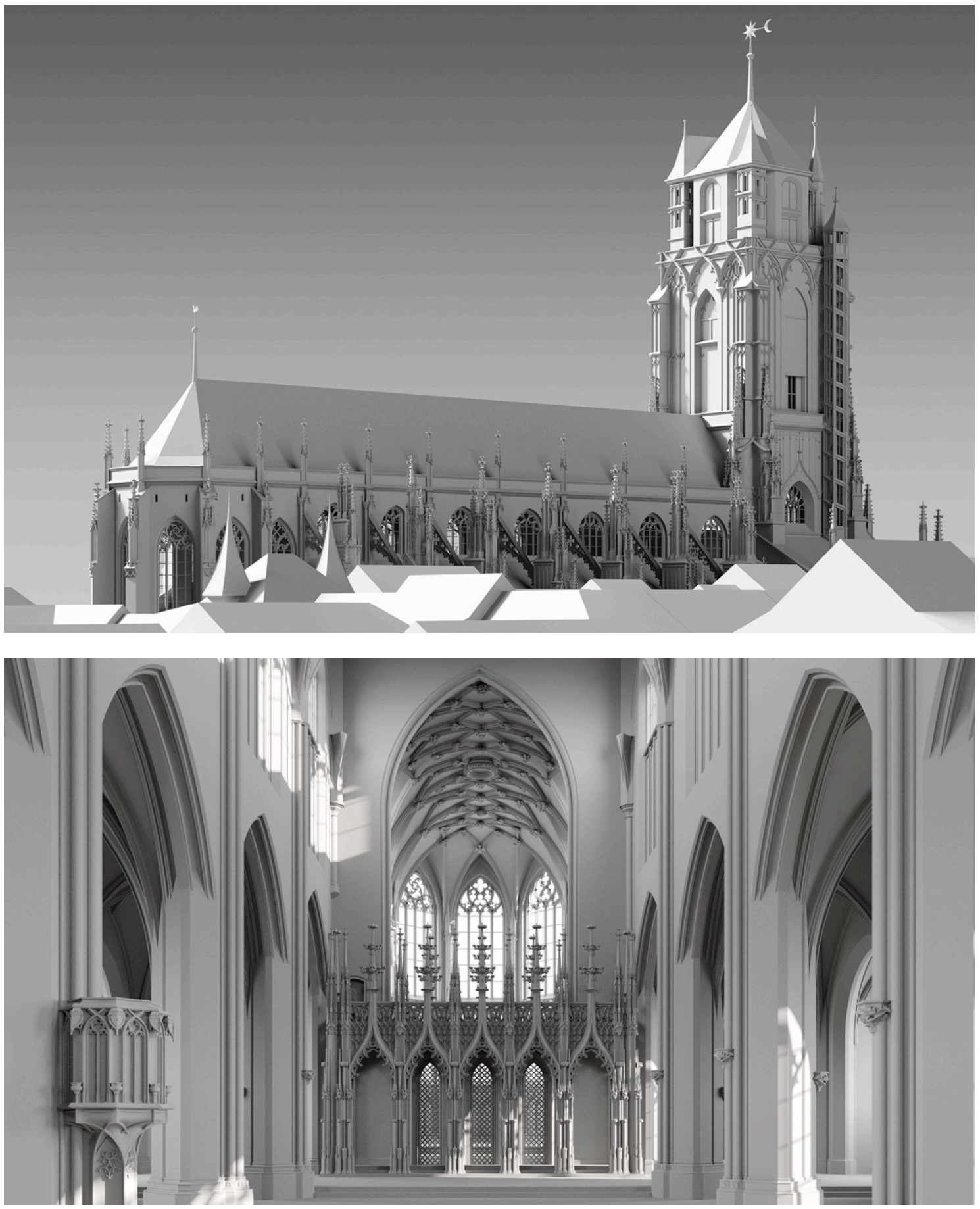

Figure 5: Berne Minster, Switzerland, for the Berne Minster Foundation.

example by means of coloured overlays, but this will always prevent an intuitive architectural impact. The primarily graphic of a coded superimposition displaces the established spatial suggestion of a purely geometric abstraction, as it has been manifested for centuries in abstract architectural models. It therefore remains a balancing act to expect that the suggestion of architecture can be mediated intuitively. The decisive element here is the architectural design of abstract geometry in virtual model making and not least its projection in virtual photography.

\section{VIRTUAL MODELLING}

Virtual modelling is the first step in the visualisation of hypotheses. In it, geometric representations of hypothetical statements about architecture are generated. The process cannot be replaced by simple algorithms, since the aim is not, for instance, to simplify the cubature of an existing building or in any other way correspond to the literal translation of abstraction as tearing away. On the contrary, the aim is to design shapes that can 
represent an idea of a building or part of a building, i.e. to suggest them as intuitively as possible.

References for this are the historical cork, wood and plaster models mentioned above, in which a reduced formal language has been established, which are reliably interpreted as architecture, especially in the context of the mediation of lost buildings.

Geometric abstraction is generally the most appropriate way of modelling hypothetical buildings, as it gives both the uncertain knowledge and the uncertainty itself. The principal possibility of misinterpreting abstract geometry as minimalist architecture is confronted with the context of the architecture to be mediated, which is generally from the pre-modern era. In classical antiquity, for example, misunderstandable forms are rarely found, and abstraction is generally correctly recognised as abstraction. This is more difficult in the Egyptian stylistic features, such as those found in the royal city of Naga in today's Sudan. The capital ornaments used here, for example, partly have the effect of abstractions of classical antique ornaments in themselves.

One method by which geometric abstraction becomes even more apparent is geometric contrast. It is then possible when next to detailed findings there are components that are of a high degree of uncertainty and therefore appear all the more abstract in direct comparison. This contrast reinforces the effect of the formal abstraction and makes the interpretation as uncertainty more suggestive.

One method of representing uncertainty that initially would seem obvious, i.e. transparency, has proved to be useless. The inherent ambivalence is only a seeming enrichment of the visual mediation. For although transparent volumes are clearly identified as being uncertain, they prevent an architectural evaluation of the spatial impact, since they can neither limit the space as unambiguously as a solid body, nor leave it as clearly unaffected as if they were not present. In this respect, the objectively stated ambivalence turns out to be a concealment that cannot be faded out subjectively and thus appears more like a non-valence. The problem presented here is not about the materiality of the represented architecture. This circumstance is also completely independent of the use of transparency for the representation of actually transparent objects such as window glass or water, as these are clearly identified through the spatial context as such.

The materiality of solid bodies is a property that can either be represented on the basis of comprehensive findings or as a result of excessive speculation. But only the overall findings within a representation, even if it is only an excerpt, allows the materiality to be represented. If the representation of materiality within a picture discontinues, then a contrast of finding to completion is created, which contradicts the intended architecture, i.e. the visualisation of the hypothetical design idea. This idea consisted of a continuous materialisation without the purely accidental fracture of the archaeological findings. The same applies to the marking of the findings, since this likewise coincidental manifestation of erosion does not follow the original design idea.

The same applies to polychromy. Fortunately, the conception of the noble white antiquity is only marginally older than its falsification. Polychromy as a phenomenon would not need to be mentioned at all if it did not have such incredible persuasive power as a narrative. But there is nothing to be gained by pointing out, in the context of conveying uncertain knowledge, that architecture and even sculpture in antiquity were polychrome. For the actual mediation of uncertain knowledge about architecture, on the other hand, polychromy is not helpful. It dominates geometry in visual terms, and this is independent of its specific design. Even more significant is the significant change in the spatial appearance with different compositions of polychromy. This circumstance means that only the elimination of polychromy makes it possible to obtain a statement about the geometry of the room that is visually close to the hypothetical statement about the geometry of the space. The polychromatic design is then left to the imagination of the viewer, which can relate to other preserved polychromatic findings.

\section{VIRTUAL PHOTOGRAPHY}

Virtual photography is the second, complementary step towards the visualisation of hypotheses. It compensates, in a way, for the abstraction of geometry by showing it as if it were built architecture.

This means that the rules of traditional architectural photography are used with the purpose of creating a spatial impression that comes close to natural vision. At the same time, misunderstandings and misinterpretations should be avoided as far as possible. It therefore implies neither technically nor artistically experimental photography, but rather the attempt to work out the essence of the building through pictorial composition. Obviously an interpretation is unavoidable. But only to the same extent as it is unavoidable in photography by any photographer, this also applies to the virtual photography of archaeological hypotheses. The author of the hypothesis is actively involved in this 
process, which further minimises the distortion of the hypothesis. An essential element in traditional architectural photography is the unambiguousness of the point of view as well as the orientation of the portrayed buildings. These important aspects are achieved in the composition by a precise orientation of the image plane and a defined standing height of the viewpoint of the camera when taking the picture.

The precise orientation of the camera is responsible for vertical building edges appearing vertical even in the image. This is due to the geometric rule that the projected images of parallels on a surface parallel to them remain parallel to each other. However, this is not just a design decision, but imitates the physiology of human eyesight.

Human eyesight is a complex process consisting of physical processes in the eye and neurological processes in the brain. The projection itself onto the ellipsoid retina depicts straight edges of space as curved lines. It is the experience of visual perception, but also of the motion through space, that allows us to perceive objects as we would mentally describe them, for instance, as rectangular. A projection on a plane, which withdraws the objects from their original spatiality and replaces them with an artificial, perspectival projection, is all the less distorted the closer it is to the mental image that is created by the real experience of space. This is all the more important the more abstract the represented geometry is, since here the references are missing which otherwise help to orientate in the picture.

Just as important, if not more important, is the unique eye level. It alone decides on the interpretation of the dimensions of a building. Although it would be possible to mediate the dimension through displayed persons, this would have the disadvantage, similar to polychromy, of raising the degree of speculation of the entire representation. Instead, a uniform eye level could also mediate reliable information about the dimension of the architecture depicted. This point is not trivial, since it still happens that visualisations, especially of virtual models, are often taken from raised viewpoints in order to provide a broader overview. The problem here is not only that the architecture depicted was neither planned nor could ever be perceived in this way, but above all that the architectural design interpretation is being misguided.

Obviously, there is also a traditional, understandable need for general information that allows an overview as a bird's eye view, similar to aerial photography. Such representations, which are common in architectural modelling, especially of urban development analyses or planning, operate less as architectural spatial impressions than as analytical representations of objects.

Consequently translated into a visualisation, this means that aerial photographs must above all be clearly distinguishable from perspectives in order not to be perceived as views that are intended to mediate an impression of space. This is achieved by two photographic parameters. On the one hand, the projection is made from a decidedly inclined viewing angle, on the other hand, from a determined large distance. Isometry fulfils both conditions, as it has a reasonably steep angle of incidence of a good 35 degrees, and at the same time, due to the parallel projection, it corresponds to a perspective projection from an infinitely distant viewpoint. Not without reason did axonometric projection experience a renaissance in classical modernism. It is thus the unambiguities that make it possible to use the means of traditional architectural photography, including axonometry, to make abstract geometry perceivable as far as possible as built architecture.

\section{REFERENCES}

Lengyel Toulouse Academic (2020) Film about the antic metropolis Pergamon.

https://www.youtube.com/LengyelToulouseAcadem ic (retrieved 17 March 2020).

Lengyel Toulouse Architects (2020) Films about The Building Phases of Cologne Cathedral and its Predecessors and Berne Minster. https://www.youtube.com/user/LengyelToulouse (retrieved 17 March 2020).

Lengyel, D. and Toulouse, C. (2016) Die digitale Visualisierung von Architektur. Deutscher Verband für Archäologie: Blickpunkt Archäologie, 2016(2), pp.91-98.

Schock-Werner, B., Lengyel, D. and Toulouse, C. (2011) Die Bauphasen des Kölner Domes und seiner Vorgängerbauten. Cologne Cathedral and preceding buildings. Verlag Kölner Dom, Köln. 